[CONTRIbUtion fRom the Havemeyer Chemical, IABORATORY, NEW York UNIVERSITY ]

\title{
HYDRATED OXALIC ACID AS AN OXIDIMETRIC STANDARD'
}

By Arthur E. Hill and Thomas M. Smith

Received December 5, 1921

\section{Introduction}

The use of hydrated oxalic acid as a standard for alkalimetry and acidity is, according to Fresenius, ${ }^{2}$ to be credited to Mohr. On account of its cheapness and the ease with which it may be freed from common impurities by crystallization, it has found a considerable use as a standard both for acidimetric and for oxidimetric analysis, and is mentioned in a large number of standard texts, including Fresenius, Mohr, Sutton, Treadwell and Morse. In the directions for its use there is usually the implication, where the statement is not direct, that it is less reliable than the best standards, and that its chief recommendation lies in its convenience rather than in any high degree of accuracy to be attained by its use.

The three directions in which a sample of hydrated oxalic acid may be expected to vary from the theoretical composition $\mathrm{H}_{2} \mathrm{C}_{2} \mathrm{O}_{4}, 2 \mathrm{H}_{2} \mathrm{O}$ are as follows: first, by the presence of salts or acids not removed during its manufacture; second, by the presence of superficial or included water; and third, by deficiencies in the amount of water of hydration, due to partial efflorescence during preparation. The first of these possibilities has received considerable attention, and the literature ${ }^{3}$ gives many slightly varying methods for the elimination of such impurities, by recrystallization from hydrochloric acid-water solution, by conversion into ethyl oxalate and subsequent hydrolysis, and by similar operations. The grades of oxalic acid upon the market to-day however render these precautions no longer necessary; we have found that c. P. oxalic acid as now prepared is sufficiently pure so that two recrystallizations give a product free from non-volatile residue and suitable for use as a standard as far as impurities other than water are concerned.

It is with respect to its water content, free or combined, that the acid shows the greatest variation from a constant composition. Methods for

1 The work includer in this paper is from the thesis presented by Thomas M. Smith as part of the requirement for the degree of Doctor of Philosophy in New York University, 1921.

2Fresenius, "Quantitative Chemical Analysis," (trans. Cohn) J. Wiley and Sons, 1912, vol. 1, p. 144.

Mohr, "Titrir-methode," F. Vieweg and Sohn, Braunschweig, 1886, p. 102.

${ }^{3}$ Mohr, Ref. 2, pp. 100-103. Thiele and Dechert, Z angew. Chem., 14, 1233 (1901). Habedanck, Z, anal. Chem., 11, 282 (1872). Stolba, Centr., 5, 119 (1874). Maumené, Compt. rend., 58. Morse, "Quantitative Analysis, Ginn and Co., 1905, p. 458. Iichty J. Phys. Chem., 11, 227 (1907). 
the removal of extraneous and included water, with the retention of the combined water, appear to have received little attention. Most frequently the procedure has consisted in drying the moist crystals between filter papers ${ }^{4}$ or in exposure to the air for a limited time. ${ }^{5}$ We have found that samples thus prepared come quite surprisingly close to the theoretical composition, usually showing an excess of water as low as 0.3 to $0.5 \%$; by a happy compensation of errors they might contain still less, but as the conditions are not reproducible, the method cannot be depended upon if a high degree of accuracy is desired.

The property of retaining included water in considerable amount is not peculiar to oxalic acid but it is well known that the phenomenon is general. Richards, in his thorough study of this property, says, ${ }^{6}$ "It is no careless exaggeration to state that in all my chemical experience I have never yet obtained crystals from any kind of solution entirely free from accidentally included mother-liquor; and I have, moreover, never found reason to believe that anyone else has. The amount of the inclosure varies from perhaps 0.01 to $0.5 \%$ of the total weight of the crystals." It is apparent therefore that the presence of included water is to be expected in any hydrated compound which may be proposed as a standard, and since from the nature of a hydrate high temperatures cannot be used to facilitate its removal, we have a source of error not easily avoided. The method which we have adopted as minimizing this error consists simply in powdering the crystals in a porcelain mortar until they pass a 100 -mesh sieve. This operation apparently breaks down very largely the cells which contain the water, and the large increase in surface permits a more speedy removal of water from the remaining unbroken cells. The crystals of oxalic acid are brittle and easily powdered; we have not been able to observe that there is any loss of water of hydration due to the heat of crushing. By this procedure the amount of included water may be quickly reduced to a very few hundredths of a per cent. as will be shown in the experimental part following.

In order to accomplish the evaporation of the superficial and included water without loss of water of combination, it is of course necessary to keep the crystals in an atmosphere in which the aqueous tension is less than that of the saturated solution and equal to or greater than the vapor tension of the hydrate. Recent measurements by Baxter and Lansing? have shown that this vapor tension is low, rising from $1.15 \mathrm{~mm}$. at $15^{\circ}$ to $2.65 \mathrm{~mm}$. at $25^{\circ}$, the usual range of laboratory temperatures. We have been able to find record of only one recommendation of a

${ }^{4}$ 'Thiele and Dechert, Mohr, Maumené, Ref. 3.

${ }^{5}$ Fresenius, Ref. 2.

' Richards, Proc. Am. Phil. Soc., 42, 28 (1903).

7 Baster and Iransing, This JourNal, 42, 419 (1920). 
desiccating agent suited for this compound: Richards, ${ }^{8}$ in his article on the rate of dehydration of crystallized salts, states "crystallized oxalic acid, so much used in making standard solutions, is best prepared by placing the powder over sulfuric acid of specific gravities 1.20 to 1.50; for the crystallized substance does not lose an essential amount of water at $22^{\circ}$ in an atmosphere containing aqueous vapor at a tension of $4 \mathrm{~mm}$.; while the aqueous vapor tension of its saturated solution is more than $15 \mathrm{~mm}$." The vapor tension of sulfuric acid of the densities recommended by Richards is about $4 \mathrm{~mm}$. to about $15 \mathrm{~mm}$. There is no doubt that this desiccating agent is suited to the purpose. It is, however, open to the objection that its vapor tension varies with the water content of the acid, so that in time the acid chosen, having become diluted with water taken up from the air, will show too great a vapor tension to accomplish its purpose. Speaking more generally, it may be stated that any desiccating agent chosen, with the exception hereafter noted, will, if in equilibrium with the oxalic acid at one temperature, be out of equilibrium at some other temperature such as may be met with in laboratory practice, since the vapor tension curves of the desiccating agent and the oxalic acid will not vary uniformly with change of temperature.

We have, accordingly, made use in our experiments of the principle that a hydrate, its next lower hydrate and water vapor form a univariant system, in which a saturated solution (represented here by the surface water or included water) must at any temperature dry up to form the hydrate only. The desiccating agent selected, a mixture of hydrated oxalic acid and anhydrous acid, is easily prepared by heating the hydrate in a large porcelain dish upon a water-bath for a few hours, which produces sufficient conversion into anhydrous compound to serve acceptably. An ordinary desiccator, fitted with a layer of this preparation an inch in depth is sufficient to dry a considerable quantity of hydrate for use as a standard. The various forms of apparatus devised for hastening the drying of the hydrate will be described below.

In principle, the method which we have found best suited to give a hydrate of constant composition consists in recrystallizing the oxalic acid from water until free from non-volatile impurities, draining, freeing from most of the superficial water by standing over partially dehydrated oxalic acid, grinding to pass a 100 -mesh sieve, and finally drying to constant weight again over the same desiccating agent. We have tested the crystals thus prepared for their oxidimetric value by titration with potassium permanganate solution.

\section{Experimental Part}

The weights used in the experimental work were calibrated according to the method of Richards; the balance used for weighing the oxalic acid or the sodium oxalate had

${ }^{8}$ Richards, Proc. Am. Acad. Arts Sci., 33, 26 (1897). 
a sensitivity sufficient to give an accuracy of $0.1 \mathrm{mg}$., and that used for weighing solutions in the weight burets gave an accuracy of $1 \mathrm{mg}$.

Sodium oxalate was used as the ultimate standard of the work, the salt being Sample No. 40 from the Bureau of Standards; large quantities of the standard were first heated to $105^{\circ}$ in an electric oven for 5 hours and kept in a desiccator over phosphorus pentoxide, from which small quantities were taken for further heating during 30 minutes immediately before use. Two permanganate solutions were prepared, one directly from a commercial analyzed "Reagent," and the second after two recrystallizations of the same material from twice distilled water. In each case the solution was filtered through washed asbestos immediately after preparation and again after an interval of some days through a Monroe platinum crucible. The water used was in all cases twice distilled, and the bottles, which were of 6 to 10 liters capacity, were of good quality glass; the solutions were protected from the action of light when not in use. The two solutions did not differ widely in their stability, as shown in the analyses of Table I.

\section{TABLE I}

\section{Standardization of Potassium Permanganate Solutions}

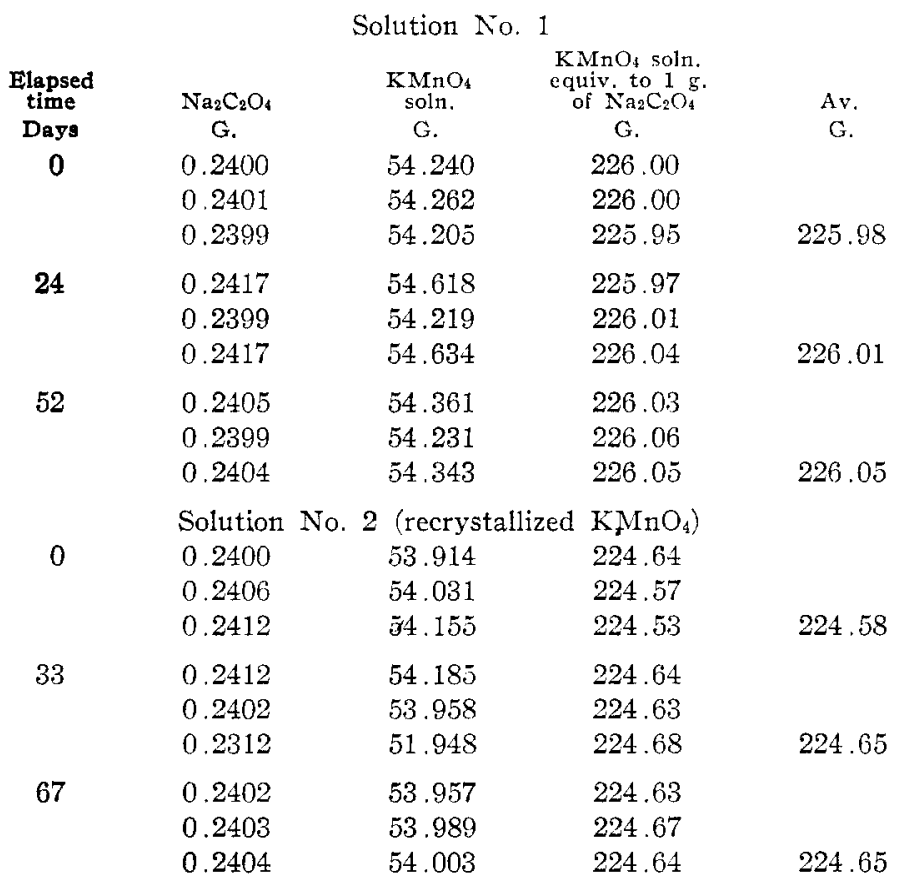

In the titrations of sodium oxalate or of oxalic acid against the permanganate, the procedure followed was that recommended by $\mathrm{McBride} ;{ }^{2}$ the solutions were kept at $70^{\circ}$ during the titrations, the acid concentration maintained constant by use of 10 cc. of $1: 1$ sulfuric acid in $250 \mathrm{cc}$. of solution, and the permanganate solution added slowly throughout. In order to minimize experimental errors, weight burets were used throughout, the greater part of the titration being performed with a fifteenth weightnormal permanganate solution, while the end-point was obtained by use of a second weight buret containing permanganate of just $1 / 10$ the concentration of the first. All

\footnotetext{
${ }^{9}$ McBride, This Journal, 34, 393 (1912).
} 
titrations were carried out by daylight, and the end-points matched with a permanent color standard made by addition of a very little cobalt nitrate to $250 \mathrm{cc}$. of water in a flask similar to those used in the titrations, the color value of which in terms of permanganate solution was determined by a series of comparisons; this value was regularly subtracted from the titration end-point. All analyses were performed in triplicate, and all analyses are included in the tables given.

In Table I are given the analyses of the two permanganate solutions which were used in the investigation, three dates being selected to cover the period of the work. The figures of Col. 1 give the elapsed time in days between the standardizations.

From the foregoing table it appears that the maximum variation in any group of three analyses is $0.11 \mathrm{~g}$., or $0.048 \%$, and the average variation $0.058 \mathrm{~g}$. or $0.025 \%$; the maximum variation from the mean is $0.06 \mathrm{~g}$. or $0.026 \%$, and the average variation from the mean is $0.033 \mathrm{~g}$.

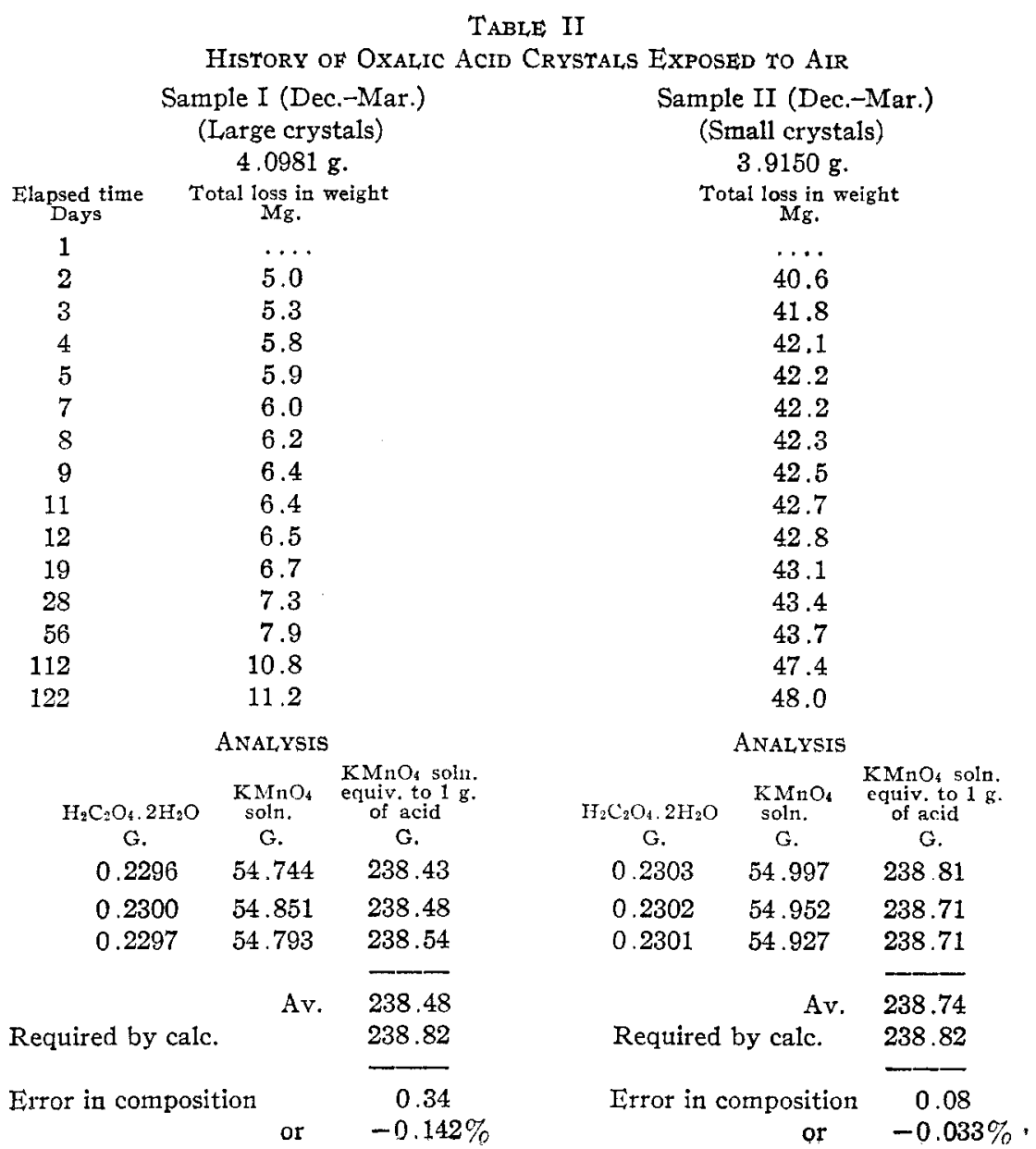




\begin{tabular}{|c|c|c|}
\hline \multicolumn{3}{|c|}{$\begin{array}{c}\text { Sample I (Aug.-Nov.) } \\
\text { (Large crystals) } \\
4.125 \mathrm{~g} .\end{array}$} \\
\hline $\begin{array}{l}\text { Elapsed time } \\
\text { Days }\end{array}$ & \multicolumn{2}{|c|}{$\begin{array}{c}\text { Total loss in weight } \\
\text { Mg. }\end{array}$} \\
\hline 1 & \multicolumn{2}{|l|}{$\ldots$} \\
\hline 2 & \multicolumn{2}{|l|}{9.6} \\
\hline 3 & \multicolumn{2}{|l|}{10.0} \\
\hline 5 & \multicolumn{2}{|l|}{10.0} \\
\hline 6 & \multicolumn{2}{|l|}{10.9} \\
\hline 8 & \multicolumn{2}{|l|}{11.1} \\
\hline 10 & \multicolumn{2}{|l|}{12.0} \\
\hline 12 & \multicolumn{2}{|l|}{12.1} \\
\hline 14 & \multicolumn{2}{|l|}{12.1} \\
\hline 21 & \multicolumn{2}{|l|}{13.2} \\
\hline 82 & \multicolumn{2}{|l|}{17.2} \\
\hline \multicolumn{3}{|c|}{ ANALYSIS } \\
\hline $\mathrm{H}_{2} \mathrm{C}_{2} \mathrm{O}_{4} \cdot 2 \mathrm{H}_{2} \mathrm{O}$ & $\begin{array}{l}\text { KMnO, } \\
\text { soln. }\end{array}$ & $\begin{array}{l}\mathrm{KMnO} \text { soln. } \\
\text { equiv. to } 1 \mathrm{~g} . \\
\text { of acid }\end{array}$ \\
\hline & & \\
\hline 0.2299 & 54.772 & 238.40 \\
\hline 0.2303 & 54.898 & 238.37 \\
\hline 0.2298 & 54.805 & 238.48 \\
\hline \multicolumn{3}{|l|}{ Required by calc. } \\
\hline Error in compos & or & $\begin{array}{c}0.40 \\
-0.163 \%\end{array}$ \\
\hline
\end{tabular}

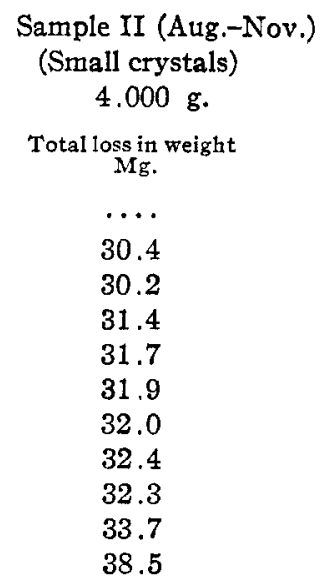

\section{ANALYSIS}

$\mathrm{H}_{2} \mathrm{C}_{2} \mathrm{O}_{4}, 2 \mathrm{H}_{2} \mathrm{O}$

G.

$\begin{array}{lll}0.2300 & 55.015 & 239.20 \\ 0.2303 & 55.098 & 239.25 \\ 0.2305 & 55.165 & 239.33\end{array}$

Av. $\quad 239.26$

Required by calc. $\quad 238.82$

Error in composition 0.44

or $\quad+0.184 \%$

or $0.015 \%$. From this it may be deduced that a series of analyses may be depended upon to about $0.02 \%$. This is also the limit of accuracy of the weighings, which may be in error by $0.05 \mathrm{mg}$. It also appears that the permanganate solutions lost in strength somewhat in a period of 2 months, the loss being $0.07 \mathrm{~g}$. or $0.031 \%$ in each case; apparently the solution made from the recrystallized salt reached equilibrium in the first month. For the purposes of comparing the oxalic acid with the sodium oxalate, discussed later, the value of the permanganate at the nearest date was used.

The oxalic acid used in the experiments was a sample of Baker's analyzed C. P. preparation, which was recrystallized three times from hot water. The material thus obtained gave no test for sulfates or chlorides, and no weighable or detectible residue upon the sublimation of a $4 \mathrm{~g}$. sample from a platinum crucible. In order to test the behavior of crystals of the size usually obtained by recrystallization, the material was treated by two different procedures in the third recrystallization; one part of the solution was surrounded by ice water, and cooled as quickly as possible, 
with stirring, so as to obtain fine crystals, while the other portion was surrounded by hot water and cooled as slowly as possible, the resulting crystals being quite large. It would seem that these two procedures should give the maximum and minimum size of crystals likely to be met with in ordinary laboratory practice.

In order to determine the rate at which these crystals lose their included water, they were exposed, after drainage, to the air of the laboratory for extended periods of time, during which they were protected from dust and kept away from laboratory fumes. The history of each sample was determined by careful weighings made at intervals beginning with the end of the first 24 hours and, finally, at the end of 3 to 4 months, by an analysis with the standard permanganate solution. The results are given in Table II.

The four samples tested, underwent a small but continuous loss of weight during periods of 3 to 4 months, when exposed to the atmospheric conditions of the winter months as well as those of late summer and early autumn. At the end of the periods of exposure, the crystals showed unmistakable evidence of efflorescence, and white stains on the watch-glasses indicated that there had also been periods of slight deliquescence. Constancy of composition cannot, it appears, be obtained by the method of direct exposure to the air. The crystals are, however, not as far from the desired composition as might be expected, the variation being at the end of the period between $+0.184 \%$ and $-0.142 \%$, as determined by analysis; the number of grams of permanganate solution required (Solution No. 2, third standardization) has been calculated by taking 224.65 as the amount required per gram of sodium oxalate, and using 134.01 and 126.048 as the molecular weights of oxalic acid and sodium oxalate respectively. The analytical data for the samples exposed in the fall have been multiplied by a factor to bring the normality of the permanganate to that used in the earlier determinations, so that comparisons may be simpler.

It may be concluded from the preceding table that crystals as ordinarily. prepared contain several tenths of a per cent. ${ }^{10}$ of included water when superficially dry (as after the first 2 or 3 days of exposure), and that expostre to air permits a gradual loss of included water and water of hydration as well, so that the composition may pass through the theoretical value (by the compensating errors of partial efforescence and small inclusion of water) to a value in which the water is less than that required by the formula.

Experiments were conducted to determine whether ordinarily prepared crystals, as described before, would come to the correct composition if

${ }^{10}$ Preliminary work done some time ago by Sister M. Augustina Brobston showed that the crystals contained from $0.30 \%$ to $0.43 \%$ of included water, as determiner by their acidimetric value compared with analyzed $\mathrm{H}_{2} \mathrm{SO}_{4}$. 
exposed to an atmosphere of the correct vapor pressure. The samples were set in ordinary desiccators over the mixture of anhydrous and hydrated oxalic acid, which will be referred to hereafter as the desiccating mixture; the results are given in Table III.

TABLE III

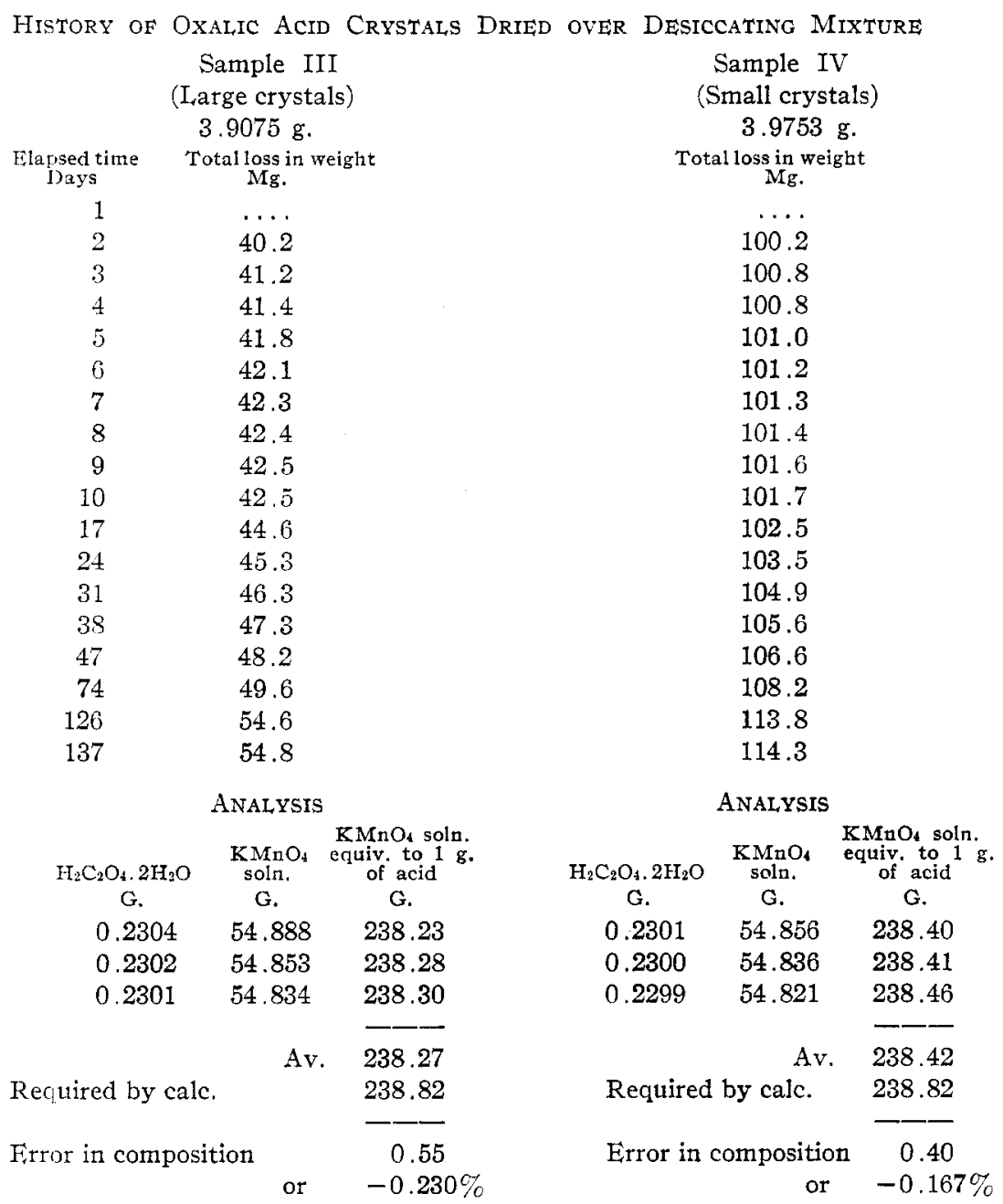

The crystals thus treated showed no evidence of efflorescence at the end of the period of 4 months, but still contained relatively large amounts of included water $(0.167$ to $0.230 \%)$. It is clear, therefore, that the material cannot be brought to a fixed and correct composition by any sort of exposure, as long as the crystals are of considerable size, and that powdering of the material is a necessity if an accurate standard is desired. 
In order to obtain a finely powdered sample for use as a standard, it was found necessary to secure a superficial dryness suitable for crushing by placing the ordinary crystals in a desiccator over the desiccating mixture; if they have been previously whirled in a centrifugal machine they become dry enough for further treatment in a day's time. They were then crushed in a glazed porcelain mortar, passed through a 100-mesh sieve of copper gauze, and tested in the several ways given below.

The simplest procedure, as regards effort, was found to consist in letting the powder attain equilibrium in the desiccator. Tests as to the effectiveness of this method are given in Table IV, in which the changes of weight have been followed over a time interval of 3 months and checked by an analysis at the end of the period. Permanganate solution No. 2, third standardization, was again used.

TABLE IV

History of Pulverized Oxalic Acid Crystals in Desiccator over Desiccatinc Mixture

Sample I

(Large crystals, ground) (Small crystals, ground) $3.6992 \mathrm{~g}$. $\quad 3.6029 \mathrm{~g}$.

Elapsed time Days

1

2

3

4

13

21

42

97

Analysis

$\mathrm{H}_{2} \mathrm{C}_{2} \mathrm{O}_{4}, 2 \mathrm{H}_{2} \mathrm{O}$
$\mathrm{G}$. $\underset{\substack{\mathrm{KMnO}_{4} \\ \text { soln. }}}{\mathrm{G} .}$

G.

0.2301

0.2299

0.2298

\subsection{9}

54.919

54.886

\section{Av. 238.86}

Required by calc.

238.82

Frror in composition

$$
\begin{array}{cc} 
& 0.04 \\
\text { or } & +0.017 \%
\end{array}
$$

loss in weight

$\mathrm{Mg}$.

i.

0.0

0.1

0.2

0.3

0.4

0.4

1. 1

$\mathrm{KMnO}_{4}$ soln. of acid

238.85

238.88

238.84 Total loss in weight

Mg.

...

0.0

0.1

0.1

0.3

0.4

0.5

1.0

\section{Analysis}

$\mathrm{H}_{2} \mathrm{C}_{2} \mathrm{O}_{4}: 2 \mathrm{H}_{2} \mathrm{O}$ $\mathrm{KMnO}_{4} \quad \mathrm{KMnO}_{4}$ soln.

$\mathrm{G}$. soln.
0.2300
0.2304
0.2302

54.940

55.032

54.995

238.87

238.85

238.90

$$
\text { Av. } 238.87
$$

Required by calc. $\quad 238.82$

$$
\begin{array}{cc} 
& -\bar{c} \\
\text { Error in composition } & 0.05 \\
\text { or } & +0.021 \%
\end{array}
$$

The crystals thus prepared vary from the theoretical composition by only about $0.02 \%$, in the direction of deficiency of water; the variation is of the same magnitude as the errors of analysis. If the loss during standing after the first day, which amounts to $1.0 \mathrm{mg}$. and $1.1 \mathrm{mg}$., respectively, be added, it appears that at the end of that period also the 
composition agrees with theory within $0.02 \%$, in the direction, however, of excess of water.

Attention was given to the problem of hastening the drying of the powder, so that the time required for the preliminary and final drying might be reduced to a matter of hours in place of the 2 days necessary when a desiccator is used. It seemed probable that that end could be secured if a current of air of correct aqueous tension could be substituted for the closed space of the desiccator. A preliminary apparatus devised for this purpose consisted of a small bell jar, in which the crystals were placed in a crystallizing dish, and the apparatus inserted into a larger jar of mercury; the neck of the bell-jar was fitted with an outlet-tube and an inlet-tube, the latter being connected with two $15 \mathrm{~cm}$. U-tubes filled with the usual desiccating agent, and the former rumning to a guard tube and thence to a water pump. When a moderately rapid stream of air was drawn throigh the apparatus, it was found that the preliminary drying could be accomplished in 30 to 60 minutes, and the final drying of the powder in 1 hour. During the course of the experiment, however, it was found that the action of the current of air for a period of 17 to 20 hours resulted in a distinct loss of water, analysis showing a deficit of $0.16 \%$ and $0.22 \%$ of water in two groups of analyses. The explanation appears to be that the aqueous tension of the air used (it being mid-winter) was too low, and that equilibrium was not attained in the rapid passage of the air through the drying tubes. To eliminate this source of error, a washbottle containing a saturated solution of oxalic acid was set at the beginning of the train; the air passing through this should take up more than the necessary amount of water, which should be reduced to the correct amount in passing through the U-tubes. The use of this device ended the apparent dehydration of the crystals. In Table $V$ are given the results obtained with the train, the first three analyses being conducted without

TABLE $V$

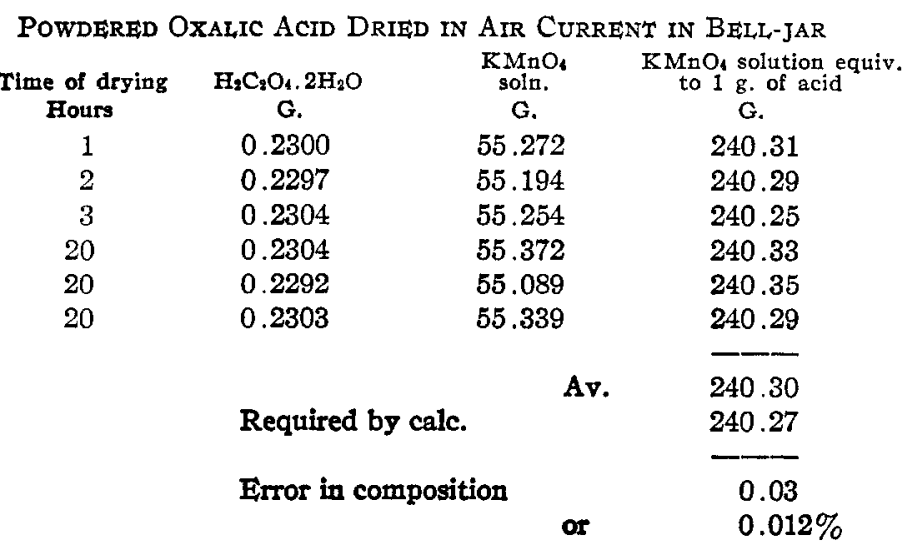


the use of the saturated solution and the second three with its use. Potassium permanganate No. 1, second standardization (see Table I), was used in the analyses.

It appears from Table $\mathrm{V}$ that the powder is completely dried in as short a time as 1 hour, and that there is no detectible change in 20 hours in a current of air properly prepared. It remained only to modify the apparatus if possible in the direction of greater simplicity and convenience. The apparatus which we have found best suited is made from a $30 \mathrm{~cm}$. length of rather wide combustion tubing, into which the crystals are inserted in a porcelain combustion boat of the usual form; the U-tube of the desiccating agent and, for safety's sake, the wash-bottle of saturated oxalic acid solution are connected as before, and the preliminary and final drying carried on for 30 minutes and 1 hour respectively. Table VI gives a series of analyses conducted in this fashion, but without the use of the saturated solution in the train. Permanganate solution No. 1, second standardization, was used in the analysis.

TABLE VI

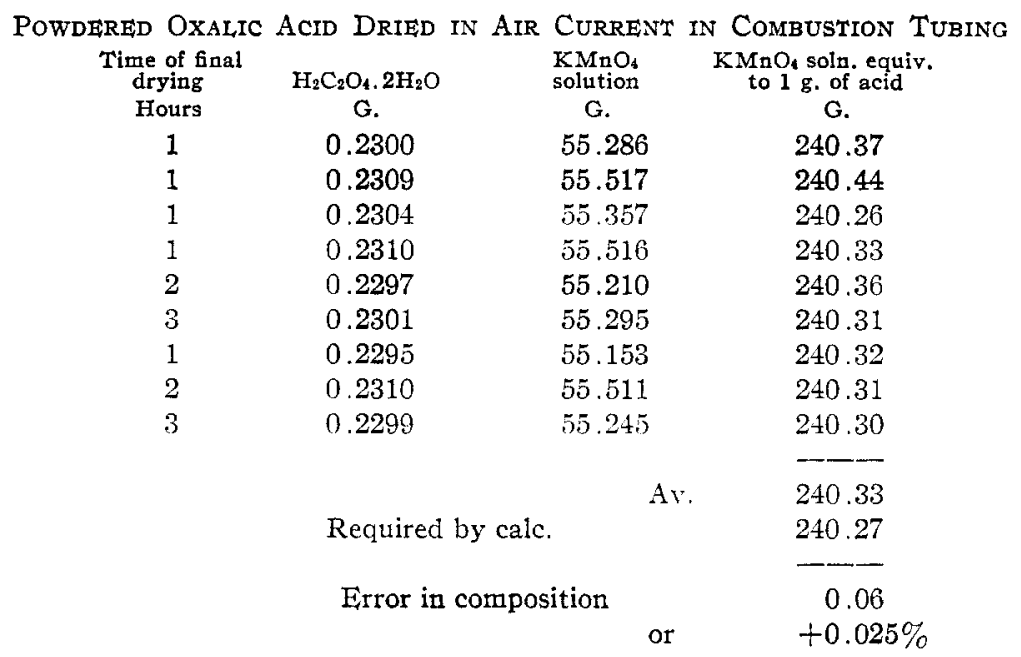

From the series of analyses obtained it may be stated that hydrated oxalic acid, as prepared above, agrees with sodium oxalate in reducing effect upon permanganate to within $0.025 \%$, the acid appearing to have a reducing value greater by that amount than the value calculated from its molecular weight. This small difference is of the same order as the error in the analysis, but from the frequency of its occurrence it does not seem to be mere chance error. It may be explained possibly as due to that amount of error in the composition of the Bureau of Standards sodium oxalate, or, as seems more probable, to the occurrence of inequalities in temperature in the desiccator during laboratory working conditions, 
which might produce a partial efforescence of the exposed hydrate. The point is left unsettled at this time.

It is our purpose to compare oxalic acid prepared in this manner with other standards used in acidimetry, and to test the same method of preparation in the study of other hydrated compounds used as standards, including Mohr's salt and copper sulfate. ${ }^{11}$

\title{
Summary
}

1. Crystals of hydrated oxalic acid as usually prepared from aqueous solution contain several tenths of a per cent. of included water, which is not wholly lost by 4 months' exposure to atmospheres of the same aqueous tension as the crystals or higher.

2. Crystals superficially dry will lose this included water in about 24 hours' time if ground to pass a 100-mesh sieve and set in an atmosphere of an aqueous tension in equilibrium with the hydrate.

3. A mixture of hydrated and anhydrous oxalic acid is the only desiccating agent giving an aqueous tension in equilibrium with the hydrate at all temperatures.

4. The powdered hydrate can be dried in about 1 hour's time in a current of air passed over this desiccating mixture, so that its oxidimetric value agrees with that of sodium oxalate within $0.025 \%$.

NEW YORK, N. Y.

[CONTRibution from the Chemistry Department, University of Washington]

\section{THE MEASUREMENT OF OVERVOLTAGE}

\author{
By Herman V. Tartar and Harmon E. Keyes \\ Received December 8, 1921
}

\section{Introduction}

It is now generally agreed that overvoltage is an electromotive force which acts counter to the applied e.m.f. during electrolysis. For the purpose of this paper hydrogen overvoltage is defined as an e.m.f. which is the difference of potential between a reversible hydrogen electrode and an electrode surface at which hydrogen gas is being formed from hydrogen ions by electrolysis, both potential measurements being referred to the same electrolyte.

Opinion has been divided as to the proper method of determining overvoltage, especially when high and varied current densities are used. Two distinct methods of measurement have been employed; (1) the commu-

11 The possible use of a mixture of hydrate and anhydrous compound as a drying agent came to the attention of one of us in conversation some 20 years ago with Prof. Robert W. Hall, then professor of analytical chemistry at New York University. It is not remembered that Professor Hall claimed originality for the idea, and we have not been able either to find its origin or to hear that it has ever been tested. 